\title{
Leishmaniasis and Tuberculosis Concurrent Infection in Humans and Animals in Kala-azar Endemic Areas of Bangladesh
}

Rahman $\mathrm{MF}^{1}$, Alam $\mathrm{MJ}^{2}$, Sarker $\mathrm{MAS}^{3}$, Uddin $\mathrm{MJ}^{4}$, Nipa $\mathrm{SA}^{5}$, Alim $\mathrm{MA}^{6}$, Matsumoto $\mathrm{Y}^{7}$, Rahman $\mathrm{MB}^{8}$ DOI: https://doi.org/10.3329/jafmc.v14i2.45882

\begin{abstract}
Introduction: Co-infection of parasitic disease and pulmonary tuberculosis are increasing public health problem especially in developing countries like Bangladesh. More than $81 \%$ of cases and deaths from TB are in developing countries and is aggravated by concurrency with parasitic diseases, where in Bangladesh suffer a substantial no of Kala-azar cases in each year. Cattle are found to have bovine tuberculosis but at the same areas of Kala-azar endemicity, whether this animal found to be positive.
\end{abstract}

Objectives: To findout the concurrent infection in humans and animals in Kala-azar endemic areas of Bangladesh.

Materials and Methods: This study was conducted at Surya Kanto Hospital, Mymensingh and four Kala-azar endemic Upazilla of Mymensingh, Tangail and Pabna District. A total of 300 highly suspected tuberculosis patients reported from Kala-azar endemic areas and 170 cattle's blood sample, 110 cattle spleen samples were included in this study. Cattle blood and spleen samples were investigated for both TB and Kalaazar and all the TB cases were investigated for Kala-azar. Buffy coat from venous blood was taken for ELISA and PCR. Spleen samples were sonicated and then were examined by PCR. All patients' blood was tested with ICT (rK39) for Visceral Leishmaniasis. ICT positive patient's splenic aspiration was examined by smear microscopy with 10x 100 magnifications. After that, all these samples were tested by ELISA and PCR.

Results: Out of total 300 patients $162(54 \%)$ were found positive for TB. Blood sample of 180 patients was tested with ICT rK39 and 12(7\%) patients were found positive for leishmaniasis who suffered from Tuberculosis. These 12(7\%) patient's splenic smear were examined of which $11(92 \%)$ of them were found positive for leishmania. But using ELISA all 12(100\%) patient smears were found positive. Out of 170 cattle blood $12(7.1 \%)$ were ICT positive indicating prevalence of bovine tuberculosis and of these 170 cattle blood 20(11.8\%) were found positive for antileishmania antibody. With PCR of these 20 , no one was found positive for Leishmania.

Conclusion: Visceral leishmaniasis and tuberculosis coinfection have drawn attention clinically. This study found the presence of leishmaniasis and tuberculosis concurrently in humans in Kala-azar endemic areas, wherein bTB antibody was detected in cattle. Though concurrently leishmania antibody was found in cattle but was not proved by PCR which requires further studies.

Key-words: Leishmania, Tuberculosis, Bovine tuberculosis, Concurrent infection, Kala-azar endemic areas.

\section{Introduction}

Co-infection of parasitic disease and pulmonary tuberculosis (TB) are increasing public health problem especially in developing countries like Bangladesh. TB and parasitic diseases in humans are infectious that exhibit an extensive distribution, causing serious harm to humans ${ }^{1,2,3}$. More than $81 \%$ of cases and deaths from TB are in developing countries and is aggravated by concurrency with parasitic diseases ${ }^{4}$. As per Directorate General Health services report ${ }^{5}$; in Bangladesh about 1068 patients suffered from Kala-azar with 3 deaths cases reported in the year 2014. El-Safi et al found in their study leishmania-tuberculosis co-infection is not uncommon in clinical practice in East Africa. In that study, they found about $66 \%$ patients were Leishmanin Skin Test (LST)-positive, $26 \%$ patients were tuberculin-positive and $20 \%$ patients were positive for both tests 6 . In India, Bangladesh and other South East Asian countries, both these infections are highly endemic and in about $20 \%$ cases co-infection of these pathogens are reported ${ }^{7}$. Since Leishmaniasis is as zoonotic as anthroponotic and in Bangladesh especially in Kala-azar endemic areas the people lives close to domestic animals even in same living room. So chance of concurrency of Leishmaniasis and

1. Maj Gen Md Fashiur Rahman, SPP, ndc, MBBS, MPH, LLB, FCGP, MBA, MSS, PhD fellow, Director General Medical Services, Ministry of Defence, Dhaka (E-mail: fashi502@yahoo.com) 2. Professor Md Jahangir Alam, MBBS, MPhil, FCGP, WHO - Fellow, Department of Community Medicine, Monno Medical College, Manikgonj 3. Dr Md Abu Sayeed Sarker, DVM, MS, PhD, Upazilla Live Stock Officer, Trishal, Mymensingh 4. Brig Gen Dr Md Jalal Uddin, MBBS, MPH, Commandant, Armed Forces Medical Institutes, Dhaka 5. Dr Shamim Ara Nipa, DVM, MS, PhD fellow, Department of Microbiology and Hygiene, Bangladesh Agricultural University, Mymensingh 6. Professor Md Abdul Alim, DVM, MS, PhD, Department of Parasitology, Bangladesh Agricultural University, Mymensingh 7. Professor Yoshitsugu Matsumoto, DVM, MSc, PhD, Laboratory of Molecular Immunology, Department of Animal Resource Sciences, Graduate School of Agricultural and Life Sciences, The University of Tokyo, Japan 8. Professor Md Bahanur Rahman, DVM, MSc, PhD, Department of Microbiology and Hygiene, Bangladesh Agricultural University, Mymensingh. 
tuberculosis in humans and animals may be there. Akter et al in their study stated, cattle are the preferred host for the sand fly vector of $V L$ in the Indian subcontinent, cattle may act as reservoir of Visceral Leishmaniasis $(\mathrm{VL})^{8}$. The epidemiology of $\mathrm{VL}$ and the possible role of cattle for $\mathrm{VL}$ transmission has not been established in Bangladesh so far. But in their study 50 cattle blood samples were examined with rk39 strip test and two samples (4\%) gave positive band, and they suggested for Polymerase Chain Reaction (PCR) to identify the parasitic $\mathrm{DNA}^{8,9}$. A study conducted by Alam et al in 2011 on cattle in Trishal Upazila, Mymensingh and they found $9.4 \% \quad(n=13)$ cattle to be positive by Enzyme Linked Immunosorbent Assay (ELISA). But no parasite DNA was detected in either Ln PCR or Loop- mediated Isothermal Amplification (LAMP) ${ }^{10}$.

In Bangladesh, from Pharo et al and Samad and Rahman it was found that the prevalence of bTB in cattle has been reported as 5.9\% and 3.05\% in Pabna and Mymensingh district respectively ${ }^{11,12}$. From another study ${ }^{13}$, the prevalence of bTB were found varied in different Upazillas; highest was found in Shahjadpur and Ullapara Upazilla (9.26\%) and lowest 5.56\% was in Kamarkhand Upazilla. In Shirajganj sadar and Belkuchi the prevalence was $7.41 \%$. Akter $S$ et al found $4 \%$ of the cattle to have positive band with rK39 strip test in Trishal study area ${ }^{8,9}$.

\section{Materials and Methods}

This descriptive cross-sectional study was conducted at Surya Kanto Hospital, a part of Mymensingh Medical College Hospital, Trishal, Fulbaria Upazilla of Mymensingh, Madhupur Upazilla of Tangail and Chatmohor of Pabna district during the period of September 2014 to September 2017. A total of 170 cattle's blood sample, 110 cattle spleen samples and 300 suspected tuberculosis patients reported from Kala-azar endemic areas were included in this study. Cattle blood and spleen samples were investigated for both TB and Kala-azar and all the TB cases were investigated for Kala-azar. Buffy coats were taken from venous blood for ELISA and PCR. Spleen samples were sonicated and then were examined by PCR. All patients' blood was tested with ICT (rK39) for Visceral Leishmaniasis. ICT positive patient's splenic aspiration was examined under a microscope with 10x100 magnification. After that, all these cases were tested by ELISA and PCR.

\section{Results}

Among the 180 TB population 122 (67.77\%) were male and $58(32.22 \%)$ were female. The male: female ratio of TB patients was 2.1: 1 with the mean age 38.83 years. Almost 158 $(87.76 \%)$ of patients both male and female were found within $16-60$ years, the productive age of life. Among 140 Kala-azar patients $77(55.0 \%)$ were male and 63(45.0\%) were female. The male: female ratio of Kala-azar (KA) patients was 1.2: 1 with the mean age 29.5 years. Almost eighty $(79.9 \%)$ percent of patients both male and female were found within $0-45$ years, in the younger age group of life. Maximum patients 43 $(30.7 \%)$ were found in the age group of $0-5$ years (Table-I). Mantoux test was for primary screening and out of admitted
180 patients 171(95\%) were found positive. Sputum smear (SS) microscopy were done for both admitted and outdoor patients and out of total 300(admitted and outdoor) 162(54\%) were found positive for TB. X-ray Chest plays important role especially for the diagnosis of pulmonary tuberculosis and out of 180 admitted patients 166(92\%) were found positive for TB. All 138 negative cases by SS were sent for GeneXpert, out of which $18(13.04 \%)$ were diagnosed as positive (Figure-I). Out of selected 160 respondents from respective study area (Community), 24(15.0\%) were found positive by rk39, of which $7(12.3 \%)$ from Fulbaria, 9(18.8\%) from Trishal, 4(13.3\%) from Madhupur, 4(16.0\%) from Chatmohor Upazilla (Figure-II). Out of 180 TB patients 28 (15.6\%) cases became positive for Leishmania with rk39 strip kits. These cases were then tested by PCR and $14(7.78 \%)$ became positive, seemed to be concurrently infected both for TB and VL (Table-II). Out of 140 suspected patients of Leishmania were first tested by Rk39(ICT) strip kit where 135 (96.4\%) were found positive for Leishmania. These 135 cases were tested with ELISA and $130(96.3 \%)$ cases were found positive. Furthermore, PCR (both $\mathrm{Ln}$ and real) was done and 124 (95.4\%) were found positive out of these 130 cases. Splenic aspiration smear test was done for all cases and all were positive for Leishmania. These 124 cases were tested with sputum smear microscopy for TBwhere $10(8.1 \%)$ cases were found positive. Afterwards GeneXpert was done for these 10 cases and all were found positive for TB seemed to have concurrent infection both with VL and TB (Figure-III). From 170 cattle 12 (7.1\%) were found ICT positive for TB and 158 (92.9\%) were found negative (Figure-IV). Over the same cattle this study tried to find out Leishmania as such blood of 170 cattle was tried with rk39 ICT kit and $20(11.8 \%)$ cattle were found positive having anti leishmania antibody. These 20 cases were tested with PCR and no cases were found positive for Leishmania (Table-III).

Table-I: Distribution of TB and Kala-azar patients by age and sex

\begin{tabular}{|l|r|r|r|r|r|r|}
\hline \multirow{2}{*}{$\begin{array}{l}\text { Age group } \\
\text { in years }\end{array}$} & \multicolumn{3}{|c|}{ TB } & \multicolumn{3}{|c|}{ Kala-azar } \\
\cline { 2 - 7 } & $\begin{array}{c}\text { Male } \\
\mathrm{n}(\%)\end{array}$ & $\begin{array}{c}\text { Female } \\
\mathrm{n}(\%)\end{array}$ & $\begin{array}{c}\text { Total } \\
\mathrm{n}(\%)\end{array}$ & $\begin{array}{c}\text { Male } \\
\mathrm{n}(\%)\end{array}$ & $\begin{array}{c}\text { Female } \\
\mathrm{n}(\%)\end{array}$ & $\begin{array}{c}\text { Total } \\
\mathrm{n}(\%)\end{array}$ \\
\hline $\mathbf{0 - 1 5}$ yrs & $07(3.9)$ & $03(1.7)$ & $10(5.6)$ & $22(15.7)$ & $21(15.0)$ & $43(30.7 \%)$ \\
\hline $\mathbf{1 5 - 3 0}$ yrs & $29(16.1)$ & $15(8.3)$ & $44(24.4 \%)$ & $22(15.7)$ & $16(11.4)$ & $38(27.1 \%)$ \\
\hline $\mathbf{3 0 - 4 5}$ yrs & $44(24.4)$ & $18(10.0)$ & $62(34.4 \%)$ & $17(12.1)$ & $14(10.0)$ & $31(22.1 \%)$ \\
\hline $\mathbf{4 5 - 6 0}$ yrs & $33(18.3)$ & $19(10.6)$ & $52(28.9 \%)$ & $08(5.7)$ & $06(4.3)$ & $14(10.0)$ \\
\hline $\mathbf{6 0 - 7 5}$ yrs & $06(3.3)$ & $02(1.1)$ & $08(4.4)$ & $05(3.6)$ & $04(2.9)$ & $09(6.4)$ \\
\hline $\mathbf{7 5 <}$ & $03(1.7)$ & $01(0.6)$ & $04(2.2)$ & $03(2.1)$ & $02(1.4)$ & $05(3.6)$ \\
\hline Total & $122(67.7 \%)$ & $58(32.3 \%)$ & $180(100)$ & $77(55.0 \%)$ & $63(45.0 \%)$ & $140(100)$ \\
\hline
\end{tabular}

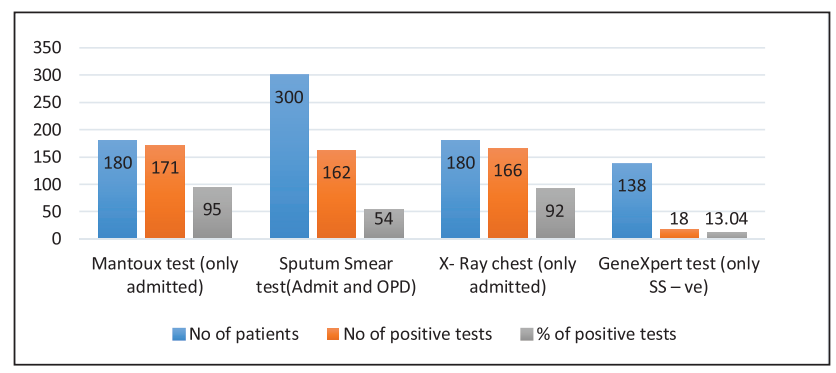

Figure-1: Distribution of TB patients by Lab tests 


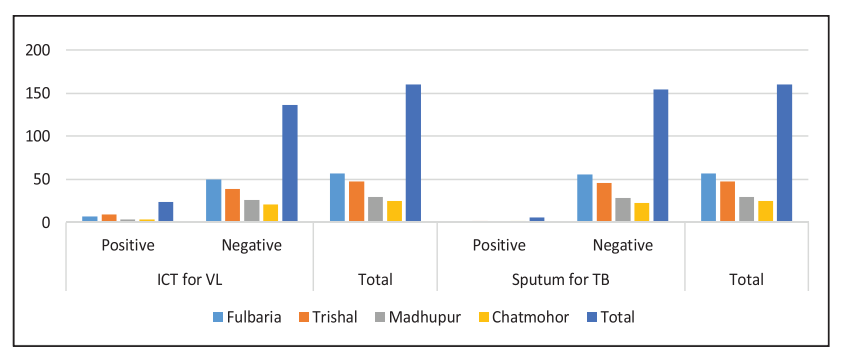

Figure-2: Community people by rK39 \& Sputum smear test

Table-II: Leishmania in TB patients admitted to SKH

\begin{tabular}{|l|c|c|}
\hline Total TB patients & ICT Rk39 Positive & PCR \\
\hline 180 & $28(15.6 \%)$ & $14(7.78 \%)$ \\
\hline
\end{tabular}

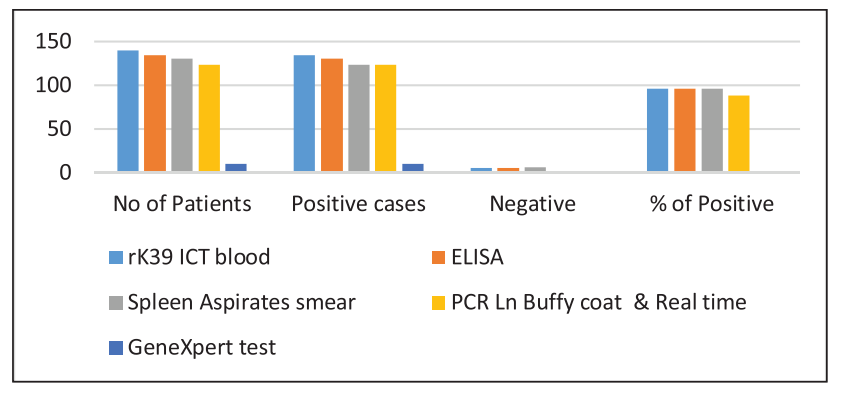

Figure-3: Leishmania \& TB from suspected Leishmania patients

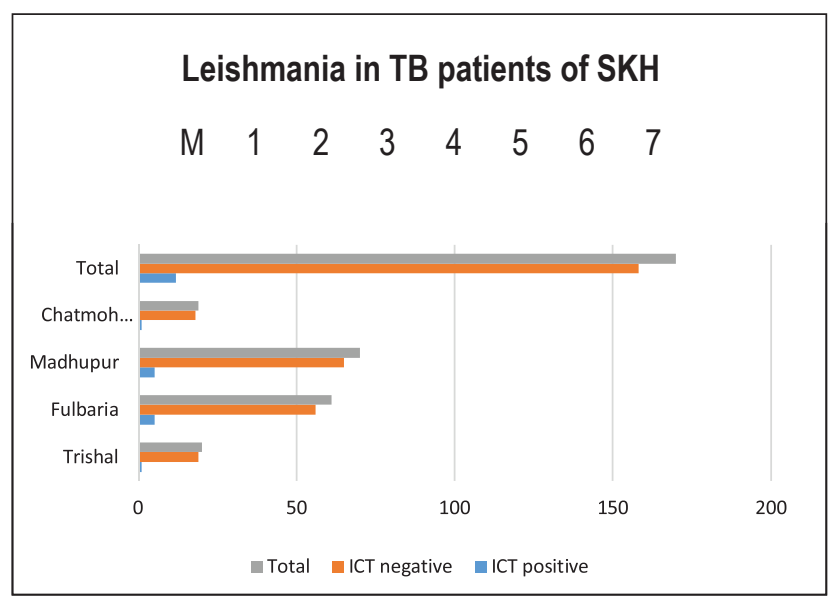

Figure-4: Distribution of Cattle by study area and ICT for TB $(n=170)$

Table-III: Detection of Leishmania from cattle by rk39 (170)

\begin{tabular}{|l|c|r|c|}
\hline Tests & No of Cattle & Positive for VL & Negative \\
\hline Rk39 ICT blood & 170 & $20(11.8 \%)$ & 150 \\
\hline PCR Ln Buffy coat & 20 & 00 & 00 \\
\hline
\end{tabular}

\section{Discussion}

This study is designed to find out concurrent infection between TB and Kala-azar in humans and animals. So the study's first concern is confirming both TB and Kala-azar then to see intertwined infection between these two diseases. Patients of TB and Kala-azar admitted in Surya Kanto Hospital,
Mymensingh where 180 TB and 140 Kala-azar patients were considered. In case of TB almost $87.76 \%$ of patients both male and female were found within 15-60 years, the productive age of life with the male female ratio of 2.1: 1 and mean age was found 38.8 years. In the study Rahman, amongst TB suspects the mean age was found 43.06 years, the male female ratio was found 2.02:114. In the study of Jermiah Seni et al, the mean age was 36.1 and male female ratio was 1.17:1 may be it was due to higher HIV positivity rate where male female equally affected and lesser elderly population ${ }^{15}$. In the study of Takele Tedesser, the mean age was found 34 years and male female ratio was 0.9:1 where it might be due to two thirds of the symptomatic sputum smear positive tuberculosis remains undiagnosed and higher HIV prevalence rate (four to six times $)^{16}$. Amongst 140 Kala-azar patients in this study 77 were male and 63 were female with the ratio of $1.22: 1$ and the mean age was 29.19 years. Maximum patients $41(31.5 \%)$ were found in the age group of $0-15$ years, in the younger age group of life. In a study, the age of the patients, out of 348 , the highest $128(36.8 \%)$ was found in $1-10$ age group and the lowest prevalence was $6(1.72 \%)$ found in 51-60 age group ${ }^{17}$. In 1995, Masum and Chowdhury reported a total of 183 chronic fever cases from different areas of Bangladesh, in which majority were in 0-15 years of age ${ }^{18}$. In Bhowmick AR, the highest prevalence of kala-azar patients was found in 1020 years age group $(67.35 \%)$ and the lowest prevalence was found in 60 and above age group $(8.33 \%)^{19}$. The prevalence of KA showed a decreased trend with the increase of age. In Bangladesh, the maximum numbers of cases were reported in the age group of $11-20$ years ${ }^{20}$.

In this study, admitted patients were tested with Mantoux test and $95 \%$ had MT positive (Figure-I) and found highly sensitive though it has false positive and false negative with some issues and cannot confirm diagnosis, it helped making initial screening and a route to diagnosis. In Islam MT Mantoux test was found more sensitive tool and $96 \%$ was found positive for $\mathrm{TB}^{21}$. In another study by Singh $\mathrm{S}$, it was found similar result of MT and was $94.3 \%$ positive for TB and was highly sensitive $^{22}$. Out of total 300 (admitted and outdoor) patients 162(54\%) were found positive for TB in this study (Figure-I) by sputum smear microscopy. Sputum smear microscopy was done for both admitted and outdoor patients, as it still remains the primary tool for diagnosing tuberculosis. Singh S stated that from 428 smear positive patients they provided repeat sputum samples and of these $71.7 \%$ were sputum smear positive $^{22}$. In another study, Khaliq A described that a total of 1000 cases of pulmonary were recruited and out of which $450(45 \%)$ were found smear positive ${ }^{23}$. Out of 180 admitted patients 166(92\%) patients were found positive for TB by X-ray Chest (Figure-I). In the study of Karim F, it was found that out of 673 Chest X-rays, 26 were found positive for tuberculosis and it was a community based study ${ }^{24}$. Shah $\mathrm{R}$ delineated in their study that they found normal CXR in 132 cases (72.13\%) and abnormal CXR was found in 51 cases $(27.87 \%)$ and was done in ENT department of a hospital25. 
In this study out of 300 patients 162 were diagnosed positive by SS microscopy (Figure-I) and 138 negative. All these negative cases were sent for GeneXpert, out of which 18(13.04\%) were diagnosed as positive. Importantly, in negative cases GeneXpert can find out positive cases if there is any undetected. Laskar $\mathrm{N}$ they found in their study that GeneXpert detected M. tuberculosis in $91(85.05 \%)$ cases $^{26}$. Aurin TH stated that M tuberculosis was detected in $283(94.33 \%)$ samples out of 300 cases $^{27}$. In Shrestha P et al, it was found that 258 smear negative cases were re-evaluated by GeneXpert of which $55\left(21.3 \%\right.$ ) were positive for $M$ tuberculosis ${ }^{28}$. As $15 \%$ (24) community people (endemic area) were found positive for leishmania, so to see the concurrent infection with TB these 24 cases were tested with Sputum smear at DOT corners of Mymensingh Medical College Hospital and 3.8\% were found positive for TB(Figure-II). In Saha P et al where 2603 population was screened for antileishmania antibody and 185 (7.11\%) were positive for antileishmania antibody but study did not investigate for TB in leishmania positive cases $^{29}$. Griensven JV stated that, although in Brazil the burden of TB and VL-HIV coinfection is substantial and TB were found to be associated with mortality in patients infected with VL. The prevalence of tuberculosis in patients infected with VL-HIV ranged from $5.7 \%$ to $29.7 \%{ }^{30}$. But in this study the TB infection with VL was $3.8 \%$. It is assumed; so far not that much research has been carried out in Bangladesh on this concurrent infection.

Surya Kanto Hospital has been selected as the prime study place of this study research, because this hospital has both TB and Kala-azar ward, where a good number of TB and Kalaazar patients stay admitted from different areas including Kalaazar endemic areas. So detecting Leishmania in TB patients and detecting TB in Kala-azar patients was the strategy to see the concurrency. During the study period 140 highly suspected kala-azar patients of Surya Kanto Hospital were persuaded for Leishmania. After trying with rK39 strip kit 135 (96.4\%) were found positive. These were tested with ELISA and 130(96.3\%) were found positive. PCR was done on 130 cases of which 124(95.4\%) were detected positive using both Ln and Real time. These confirmed Leishmania cases were examined with smear microscopy and 10(8.1\%) were found positive. After wards GeneXpert were tried with these 10 cases and all were found positive. This study surveyed 160 local people from 110 households of study area besides its survey on patients of Surya Kanto Hospital. Respondents blood were tested with rK39 strip (ICT) and 24(15\%) were found positive for leishmania antibody and it was highest (18.8\%) in Trishal and lowest (12.3\%) in Fulbaria (Figure - II). Saha P mentioned in their study that a total of 185 individuals were positive for antileishmanial antibody, of them 96 had a history of kala-azar/ PKDL and 89 (3.56\%) without any history of kala-azar (KA). The ratio of asymptomatic infection to incidence active $\mathrm{VL}$ cases was 7.9:1 at that time of their study ${ }^{29}$. Singh AS stated in their study that Of 150 asymptomatic contacts tested for IgG anti-rK39 antibodies, 55(36.6\%) were found to be positive by microwell ELISA as well as the dipstick test. Fifteen (10\% of the total number tested) were found to be positive for $\lg A$ anti-rK39 antibodies. In Bern $\mathrm{C}$ et al it was found that out of total endemic population $6.8 \%$ had leishmania antibody. It is evident that a substantial number of healthy population is found leishmania antibody positive from which certain percentages becomes sick and rest remains asymptomatic, as was found in this study.

As $15 \%$ (24) community people were found positive for leishmania so to see the concurrent infection with TB these 24 cases were tested with Sputum smear test at DOT corners of Mymensingh Medical College Hospital and 3.8\% were found positive for TB. This concurrent infection was found highest $8 \%$ in chatmohor area and $4.2 \%$ were in Trishal (Figure-II). In Saha $\mathrm{P}$ et al where 2603 population was screened for antileishmania antibody and $185(7.11 \%)$ were positive for antileishmania antibody but study did not investigate for TB in leishmania positive case ${ }^{29}$. Griensven JV stated that, although in Brazil the burden of TB and VL-HIV coinfection is substantial and TB were found to be associated with mortality in patients infected with VL. The prevalence of tuberculosis in patients infected with VL-HIV ranged from $5.7 \%$ to $29.7 \%{ }^{30}$. But in this study the TB infection with VL was $3.8 \%$. It is assumed that so far not that much research has been carried out in Bangladesh on this concurrent infection. This study tried with cattle blood to see the presence of Leishmania in the blood whether it acts as reservoir host. Out of 170 cattle blood $11.8 \%$ (20) were found positive for anti leishmanai antibody with rK39 ICT kit. All these 20 cases were tested with PCR but no one was positive for leishmania DNA (Table-III). In the study of Niti S, it was found that Antibodies to rK39 indicative of VL were detected in animals, including 1 of 161 cattle, were positive by rK39 serology. Leishmania donovani DNA was detected by PCR in 20 rK39 positive blood samples including 1 sample from a cow. May be the Leishmania DNA detected persists for a short time and was indicative of recent infection ${ }^{31}$. In the study of Alam MS, they found $9.4 \%(n=13)$ of the cattle were found to be positive by ELISA for anti leishmania antibody. Of the 13 ELISApositive cattle, only four (30.8\%) were positive in DAT. Parasite DNA was not detected in either of the molecular assays ( $L n$ PCR and LAMP) $)^{10}$. In the study Akter S, 10(1) it was found that 50 cattle blood samples were examined with rK39 strip kit and two samples (4.0\%) gave positive band ${ }^{8,9}$. Bhattarai et al found Leishmania infections among persons $(6.1 \%)$, cows $(5 \%)$, buffaloes (4\%), and goats (16\%) by using PCR.

There is no available data about the investigation of domestic cattle to identify them as reservoir host of VL in Bangladesh ${ }^{32}$. In a more recent study in Nepal, Leishmania DNA was found in several domestic animals such as goats, cows and buffaloes from an endemic area several months after the active transmission season ${ }^{32}$ but there was no evidence of Leishmania DNA in domestic cattle in Bangladesh, although the cattle were seropositive for leishmaniasis ${ }^{10}$. From this study it may be assumed that the cattle may not harbour 
leishmania for acting as reservoir host. However, it may require further studies. An overall prevalence of bTB was found in the study area was 7.1\%, detected by Antigen Rapid bTB Ab test kit. But it was found to vary in different Upazillas. Which was highest in Fulbaria (8.2\%) there after 7.1\% from Madhupur followed by $5 \%$ both in Trishal and Chatmohor Upazilla (Figure-IV). Bovine tuberculosis (bTB) is considered as one of the important zoonotic diseases prevalent widely known to exist all over the globe including Bangladesh ${ }^{13}$. From Pharo et al and Samad and Rahman, it was found that the prevalence of bTB in cattle has been reported as $5.9 \%$ and $3.05 \%$ in Pabna and Mymensingh district respectively ${ }^{11,12}$. From a study, the prevalence of bTB were found varied in different Upazillas. The highest was found in Shahjadpur and Ullapara Upazilla (9.26\%) and lowest $5.56 \%$ was found in Kamarkhand Upazilla. In Shirajganj sadar and Belkuchi the prevalence was $7.41 \%^{13}$. Akter $\mathrm{S}$ et al found in their study that $4 \%$ of the cattle to have positive band with rK39 strip test in Trishal area 8,9 .

\section{Conclusion}

This study has been able to find out a certain percentage of leishmaniasis amongst tuberculosis patients and a certain percentages of tuberculosis in leishmania patients. May be both the diseases has its predilection for concurrent infection to each other especially in humans. However endeavour had been made in this study to see the presence of leishmania in cattle. AsbTB is a proven zoonotic disease it does not require any further proof, though this study had done rapid ab test kit test for TB and antibody was detected. In case of Leishmaniasis, though few cattle were found positive for leishmania antibody but it was not proved by PCR, as such this study could not prove cattle as reservoir host. It may require more and more studies. As such this has been an important message to clinicians and veterinarians as well as researchers for enduring proper attention to manage both diseases. Mentionable here is that an effective bridging and collaboration is also seemed to be essential between human doctors and veterinary doctors especially in Bangladesh.

\section{References}

1. UNDP, World Bank. WHO Special Programme for Research and Training in Tropical Diseases (TDR): Tropical Disease Research: Progress 1999-2000.

2. World Health Organization Press; Geneva, Switzerland: 2001. WHO/TDR/ GEN/01.5.

3. Xin-Xu Li, Xiao-Nong Zhou. Co-infection of Tuberculosis and parasitic diseases in humas: A systemic review. Parasite \& Vectors 2013; $6: 79$

4. Dye C, Williams B. The population dynamics and control of tuberculosis. Science 2010; 328:856-61.

5. DGHS report. National Kala-azar Elimination Programme(NKEP), National Guideline for Kala-azar case management 2016, 3rd ed.
6. El-Safi SH, Hamid N, Omer A et al. Infection rates with Leishmania donovani and Mycobacterium tuberculosis in a village in eastern Sudan. Trop Med Int Health 2004; 9(12):1305-11.

7. Dev A, Kumar U, Sharma $P$ et al. Immunogenicity of candidate Chimeric DNA vaccine against tuberculosis and Leishmaniasis. Vaccine 2009; 27(37):5152-60.

8. Akter S, Alam MZ, Islam MT et al. Seroepidemiological study of visceral leishmaniasis and cattle as a possible reservoir host at Trishal Upazila in Bangladesh. J Bangladsh Agril Univ 2012; 10(1):79-86.

9. Akter S, Alam MZ, Islam MT et al. Seroepidemiological study of visceral Leishmaniasis and its reservoir host in Trishal Mymensingh. BSVER ASCon BAU Mymensingh 2012; 18(37):61.

10. Alam MS, Ghosh D, Khan MGM et al. Survey of domestic cattle for anti-leishmania antibodies and Leishmania DNA in a visceral leishmaniasis endemic area of Bangladesh. BMC Veterinary Research 2011; 7:27.

11. Pharo HJ, Motalib A, Routledge SF et al. The prevalence of bovine tuberculosis in the Bangladesh cattle development project. Bangladesh Veterinary Journal 1981; 15:53-6.

12. Samad MA, Rahman MS. Incidence of bovine tuberculosis and its effect on certain blood indices in dairy cattle of Bangladesh. Indian Journal of Dairy Science 1986; 39:3-6.

13. Mahmud MAA, Belal SMSH, Shoshe NZ. Prevalence of bovine tuberculosis in cattle in the selected Upozilla of Sirajganj district in Bangladesh. Bangl J Vet Med 2014; 12(2):141-5.

14. Rahman MF, Alam MJ, Uddin MJ et al. Smear Positive Tuberculosis amongst Suspects Reported to DOTS Corner of Mymensingh Medical College Hospital, Bangladesh. Mymensingh Med J 2015; 24(2):263-8.

15. Seni J, Kidenya BR, Obassy E et al. Low sputum smear positive tuberculosis suspects in a tertiary hospital in Mwanza, Tanzania. Tanzania Journal of Health Research 2012: 14(2).

16. Tedesser T, Demissie M, Berhane $Y$ et al. Yigzaw Kebede, Markos Abebe. Two-thirds of smear positive tuberculosis cases in the community were undiagnosed in Northwest Ethiopia: Population based cross sectional study. Plos One 2011, 6(12):1-6.

17. Hasan MS, Khanum H, Alam MS et al. Occurrence of KalaAzar in accordance with socio-economic conditions and associated disorders among the inhabitants of Trishal Thana, Mymensingh. Univ J Zool Rajshahi Univ 2013; 32:1-8.

18. Masum MA, Chowdhury MS, Ahmed RU et al. Outbreak of Visceral Leishmaniasis in Thakurgaon, Bangladesh. Journal of Preventive and Social Medicine 1995; 2(4-9):38-44.

19. Bhowmick $A R$ and Khanum $H$. Prevalence of visceral leishmaniasis, risk factors and associated disorders: Knowledge of inhabitants and professionals in fulbaria, mymensingh. Bangladesh J Zool 2017; 45(1):73-83.

20. Thakur CP. Epidemiological, Clinical and Therapeutic features of Bihar Kala-azar (including post kala-azar dermal leishmaniasis). Trans R Soc Trop Med Hyg 1984; 78:391-8. 
21. Islam MT, Rabbi F, Fersous $S$ et al. Evaluation of three immunological tests for the diagnosis of pulmonary tuberculosis in a rural endemic area of Bangladesh. International Journal of Mycobacteriology. Elsevier March 2014; 3:88-93.

22. Singh S, Singh J, Kumar S et al. Poor Performance of Serological Tests in the Diagnosis of Pulmonary Tuberculosis: Evidence from a Contact Tracing Field Study. PLoS ONE 2012; 7(7):e40213.

23. Khaliq A, Khan IH, Akhtar MW et al. Environmental Risk Factors and Social Determinants of Pulmonary Tuberculosis in Pakistan. Epidemiology (sunnyvale) 2015; 5(3):1-9.

24. Karim F, Ahmed J, Islam QS et al. Occupational Pulmonary Tuberculosis among BRAC Community Health Workers of Trishal, Bangladesh. BRAC Research and Evaluation Divison (RED) 2011: Research Monograph Series No 50:1-39.

25. Shah R, Talukdar DC, Fakir MAY et al. Role of chest x-ray in tuberculous cervical lymphadenitis. J Dhaka Med Coll 2013; 22(2):125-8.

26. Laskar N, Hossain MA, Fardows J et al. GeneXpert MTB/RIF Assay for Rapid Identification of Mycobacterium Tuberculosis and Rifampicin Resistance Directly from Sputum Sample. Journal of Enam Medical College 2017; 7(2):86-9.
27. Aurin TH, Munshi SK, Kamal SMM et al. Molecular Approaches for Detection of the Multi-Drug Resistant Tuberculosis (MDR-TB) in Bangladesh. PLoS ONE 2014; 9(6):e99810.

28. Shrestha P, Arjyal A, Caws M et al. The Application of GeneXpert MTB/RIF for Smear-Negative TB Diagnosis as a Fee-Paying Service at a South Asian General Hospital. Tuberculosis Research and Treatment 2015, Article ID 102430, 6 pages.

29. Saha P, Ganguly S, Chatterjee M et al. Asymptomatic leishmaniasis in kala-azar endemic areas of Malda district, West Bengal, India. PLoS Negl Trop Dis 2017; 11(2):e0005391.

30. Griensven JV, Mohammed R, Ritmeijer K et al. Tuberculosis in Visceral Leishmaniasis-Human Immunodeficiency Virus Coinfection: An Evidence Gap in Improving Patient Outcomes? Open Forum Infect Dis 2018; 5(4):ofy059.

31. Niti S, Jyotsna M, Ram S, Sarman $S$ et al. Animal reservoirs of Visceral Leishmaniasis in India. Journal of Parasitology 2013; 99(1):64-7.

32. Bhattarai NR, Auwera GVD, Rijal $S$ et al. Domestic Animals and Epidemiology of Visceral Leishmaniasis, Nepal. Emerging Infectious Diseases 2010; 16(2):231-7. 